\title{
De Garengeot Hernia: Case Report and Review of the Literature of a Rare Femoral Hernia
}

\author{
Leo Licari ${ }^{1}$ (D) $\cdot$ Sofia Campanella ${ }^{1} \cdot$ Claudia Carolla $^{1} \cdot$ Simona Viola $^{2} \cdot$ Giuseppe Salamone $^{1}$
}

Received: 16 November 2020 / Accepted: 2 February 2021 / Published online: 10 February 2021

(C) The Author(s) 2021

\begin{abstract}
De Garengeot hernia is a rare femoral hernia defined as the presence of the appendix within the femoral hernia sac. The incidence of appendicitis in this type of hernia is a rare condition that accounts for $0.08-0.13 \%$ of all De Garengeot hernias. We describe the case of a 61-year-old woman that presented at the emergency department with a tender mass (diameter $10 \times 8 \mathrm{~cm}$ ) in the right groin region for 5 days associated with pain in lower right abdomen and accompanied by fever $\left(38^{\circ} \mathrm{C}\right)$. Computed tomography (CT) of the abdomen revealed the presence of a complex fluid collection with small foci of air in the right inguinal region, measuring $9 \times 7 \times 10 \mathrm{~cm}$ in the 3 orthogonal dimensions and a blind ending tubular structure extending from the caecal base into the groin mass through a narrowed neck defect, medial to the common femoral vessels. The CT scan demonstrated the suspected diagnosis of De Garengeot hernia complicated by acute appendicitis. The intra-operative findings confirmed the diagnosis. The patient was successfully managed operatively. The surgical strategy took into account the need to significantly reduce the diffusion of the infection inside the abdominal cavity. The patient was discharged on the fifth postoperative day without peri-/ postoperative complications.
\end{abstract}

Keywords De Garengeot hernia $\cdot$ Rare hernia $\cdot$ Emergency surgery

\section{Introduction}

De Garengeot hernia is a rare femoral hernia; it is defined as the presence of the appendix within the femoral hernia sac. It accounts for $0.5-5 \%$ of all femoral hernias, it is more common in women (6:1) and it mainly affects the elderly population. The incidence of appendicitis in this type of hernia is as low as $0.08-0.13 \%$ of all De Garengeot hernias [1-2].

It was first described by a French surgeon, Rene Jacques Croissant De Garengeot, in 1731 [3].

The clinical presentation is similar to an acutely incarcerated or strangulated femoral hernia. Patients with clinical

Leo Licari

lele.licari@gmail.com

1 Department of Surgical, Oncological and Oral Sciences, University of Palermo, Via Liborio Giuffrè, 5, 90127 Palermo, Italy

2 Department of Biological, Chemical and Pharmaceutical Sciences and Technologies (STEBICEF), University of Palermo, Viale delle Scienze, Ed. 16, 90128 Palermo, Italy suspicion of femoral hernia should undergo computed tomography (CT) to confirm the diagnosis [4-5].

\section{Case Presentation}

A 61-year-old woman presented at the emergency department with a swelling (diameter $10 \times 8 \mathrm{~cm}$ ) in the right groin region for 5 days associated with pain in lower right abdomen and accompanied by fever $\left(38{ }^{\circ} \mathrm{C}\right)$. She was a nonsmoker woman with no comorbidities; she never underwent surgical operations in the past. On examination, there was a mass on the medial side of the right groin; the skin over the mass was ulcerated and with signs of inflammation (Fig. 1). By palpating, the mass was tender. The rest of the abdominal examination was unremarkable; she had no nausea and vomiting and there was no sign of bowel obstruction or peritonitis.

Laboratory data showed the white blood cell (WBC) count of $14.79 \times 10^{3} / \mu \mathrm{L}$, with $82.3 \%$ neutrophils and C-reactive protein of $48.35 \mathrm{mg} / \mathrm{L}$. The clinical suspicion of femoral abscess of unknown origin was raised up. 


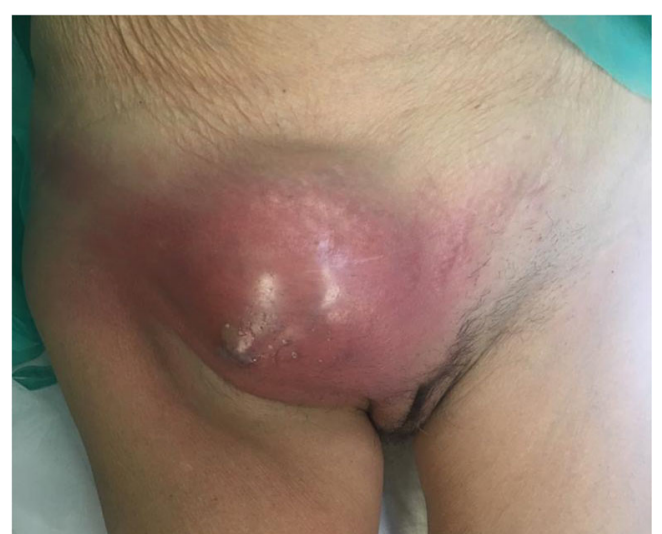

Fig. 1 De Garengeot's hernia

The CT scan of the abdomen (Fig. 2) revealed the herniation of the appendix in the femoral hernia sac. The patient received intravenous fluids and antibiotics (500 $\mathrm{mg}$ of metronidazole and $1 \mathrm{~g}$ of cefazolin); the surgical operation was proposed.

The surgical approach adopted was a combined access to the abdominal cavity and to the femoral canal through two different incisions, respectively a lower midline laparotomy and a standard oblique infra-inguinal incision under general anaesthesia. The small bowel and the colon were macroscopically undamaged. The appendix had herniated through the femoral ring into a hernia sac. Due to the small size of the defect, the sac and its contents were unable to be reduced into the abdomen. The hernia sac contained the appendix and purulent fluid. After an accurate drainage, the appendix was isolated and removed through the abdominal cavity and the hernia was repaired with herniorrhaphy without the use of a mesh, in view of the intense inflammation and presence of contaminated field. The wounds were closed in layers with sutures for deep subcutaneous tissues and skin. The antibiotic therapy was administered intravenously for the next 4 days.
Postoperative course was without complications, and she was discharged on the fifth postoperative day. No peri-/postoperative complications occurred. The histopathological findings were serosal and subserosal inflammation and fibroblastic proliferation in the appendix along with femoral hernia sac contents, concluding for acute phlegmonous appendicitis.

\section{Discussion}

The femoral hernia represents an uncommon cause of groin mass, which accounts for $3-5 \%$ of all abdominal hernias. A hernia sac can contain any of the intra-abdominal contents. Femoral hernia containing appendix accounts for only 0.5 $5 \%$ of all femoral hernias [6-8].

The differential diagnosis for uncomplicated cases includes, in addition to inguinal hernia, adnexal diseases, a varix node, ectasia of the Vena saphena magna, lipomas or other soft tissue tumours, lymphomas and hypostatic abscesses in retroperitoneal processes. In the presented case, the patient had already been diagnosed with De Garengeot hernia after having CT scan.

The CT scan is the modality of choice in the diagnosis of the De Garengeot hernia with a $100 \%$ sensitivity and $98.9 \%$ specificity [4-5]. CT scan of the abdomen can provide secure preoperative diagnosis and it remains the best way to guide the surgical approach.

The double incision approach was justified by (1) the intent to limit the infection out of the abdominal cavity; (2) the intent to limit the widening of the hernia ring that it was required to correctly expose the base of the cecum; (3) the intent to completely remove the appendix, cutting it near the base of the cecum, in order to avoid further stump appendicitis. We

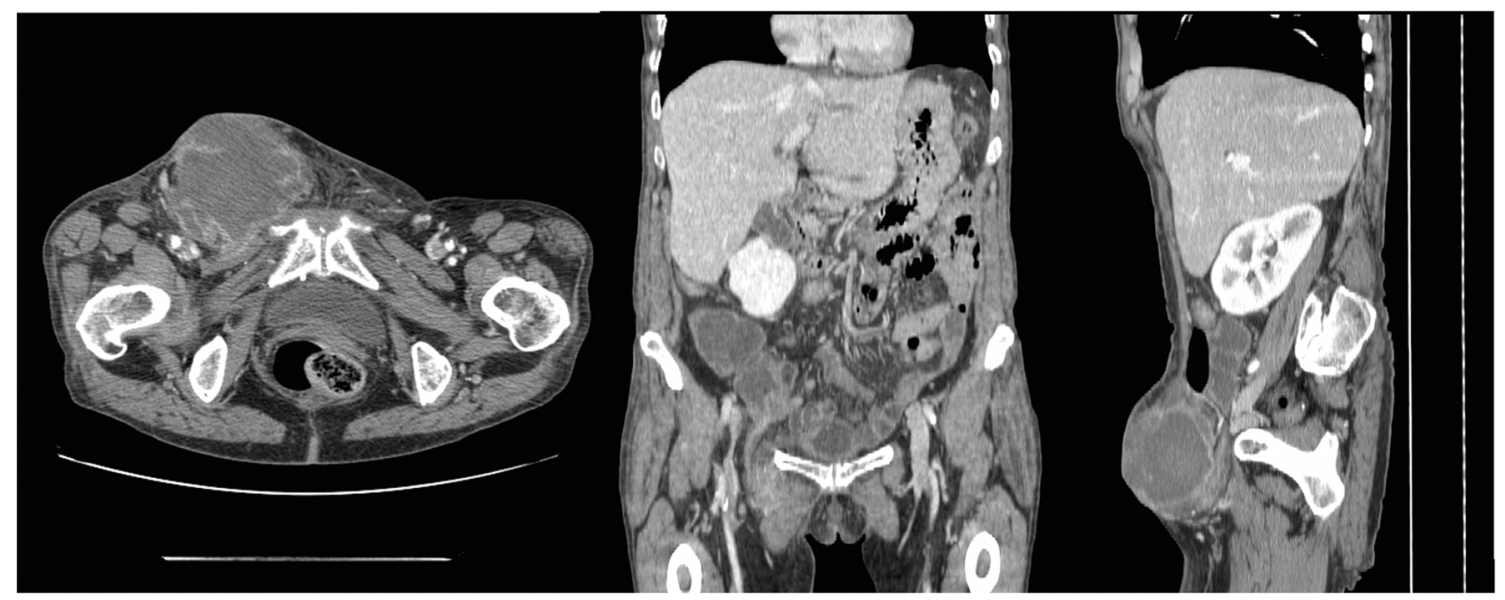

Fig. 2 Axial, coronal, and sagittal computed tomography (CT) showing De Garengeot hernia. Description: presence of a complex fluid collection with small foci of air in the right inguinal region, measuring $9 \times 7 \times 10 \mathrm{~cm}$ in the 3 orthogonal dimensions and a blind ending tubular structure extending from the caecal base into the groin mass through a narrowed neck defect, medial to the common femoral vessels 
suggest the double incision approach in the rare cases of De Garengeot hernia in the setting of contaminated/dirty field.

Due to the rarity of this condition, there is no specific guideline as for the surgical procedure. We propose (1) to consider rare femoral hernia when a tender mass is present in the femoral region; (2) if suspicion of rare hernia arises, consider to perform CT scan of the abdomen in order to make a precise preoperative diagnosis; (3) if it is confirmed the diagnosis of rare complicated femoral hernia, please consider a surgical approach that prevent contamination of the abdominal cavity; (4) complicated femoral hernia remains a surgical emergency.

Acknowledgements The authors want to thank the Dr. Massimiliano Lenzo (Department of Biomedicine, Neuroscience and Advanced Diagnostics, University of Palermo, 90127, Palermo, Italy) who provided data about the radiological findings contributing editing the related part in the manuscript.

Authors' Contribution Sofia Campanella, Leo Licari, Claudia Carolla and Simona Viola collected the data and wrote the manuscript; Leo Licari, Simona Viola and Giuseppe Salamone reviewed the literature and the final manuscript. All authors read and approved the final version of the manuscript.

Funding Open access funding provided by Università degli Studi di Palermo within the CRUI-CARE Agreement.

Data Availability Refer to corresponding author.

\section{Declarations}

\section{Consent for Publication Obtained.}

Competing Interests The authors declare no competing interest.

Open Access This article is licensed under a Creative Commons Attribution 4.0 International License, which permits use, sharing, adaptation, distribution and reproduction in any medium or format, as long as you give appropriate credit to the original author(s) and the source, provide a link to the Creative Commons licence, and indicate if changes were made. The images or other third party material in this article are included in the article's Creative Commons licence, unless indicated otherwise in a credit line to the material. If material is not included in the article's Creative Commons licence and your intended use is not permitted by statutory regulation or exceeds the permitted use, you will need to obtain permission directly from the copyright holder. To view a copy of this licence, visit http://creativecommons.org/licenses/by/4.0/.

\section{References}

1. Salkade PR, Chung AY, Law YM (2012) De Garengeot's hernia: an unusual right groin mass due to acute appendicitis in an incarcerated femoral hernia. Hong Kong Med J 18(5):442-445

2. Bustamante Recuenco C, García-Quijada García J, Cendrero Martín M, Carabias Hernández A, Serantes Gómez A, Sanz Muñoz P, Delgado Millán MÁ, Jover Navalón JM (2019) De Garengeot's hernia: case report and literature review. Int J Surg Case Rep 64:58-61. https://doi.org/10.1016/j.ijscr.2019.09.037

3. Garcia-Amador C, De la Plaza R, Arteaga V, Lopez-Marcano A, Ramia J (2016) Garengeot's hernia: two case reports with CT diagnosis and literature review. Open Med (Wars) 11:343-349

4. Ikram S, Kaleem A, Satyapal D, Ahmad SM (2018) De Garengeot's hernia: a rare presentation of the wandering appendix. BMJ Case Rep 2018:bcr2017223605

5. Salamone G, Licari L, Atzeni J, Tutino R, Gulotta G (2014) Histologic considerations about a rare case of recurrent incisional hernia on McBurney incision. Ann Ital Chir 85(ePub): S2239253X14022828

6. O'Connor A, Asaad P (2019, 2019) De Garengeot's hernia with appendicitis-a rare cause of an acutely painful groin swelling. J Surg Case Rep (5):rjz142. https://doi.org/10.1093/jscr/rjz142

7. Bidarmaghz B, Borrowdale RC, Raufian K (2018) A rare presentation of appendicitis inside the femoral canal: case report and literature review. Surg Case Rep 4(1):143. https://doi.org/10.1186/s40792018-0552-y

8. Guercio G, Augello G, Licari L, Dafnomili A, Raspanti C, Bagarella N, Falco N, Rotolo G, Fontana T, Porello C, Gulotta G (2016) Acute appendicitis: should the laparoscopic approach be proposed as the gold standard? Six-year experience in an Emergency Surgery Unit. G Chir 37(4):174-179

Publisher's Note Springer Nature remains neutral with regard to jurisdictional claims in published maps and institutional affiliations. 